\title{
Design Simulation of IEEE 802.11a Orthogonal Frequency Division Multiplexing (OFDM) Modem
}

\author{
Husam Eldin A.Rahman ${ }^{1}$, Dr. Mohammed Ali Hammad Abbas ${ }^{2}$
}

\begin{abstract}
This work is concerned with the design, software implementation considerations and performance evaluation of an 802.11a WLAN modem at base band level. The function of each block in transmitter is completely specified in the standard. But the receiver is not fully specified. Only the broad functionalities and the performance requirements are specified. Hence design of a receiver is a challenging task.
\end{abstract}

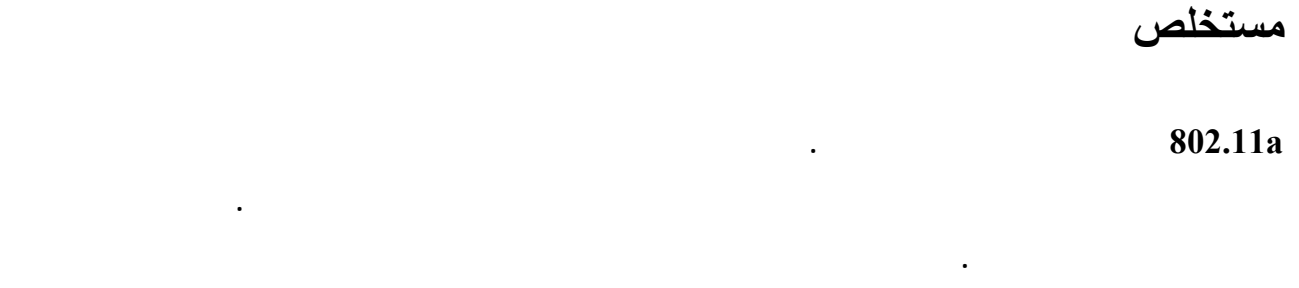

\section{Introduction}

Wireless Local Area Networks (WLAN) are evolving towards the development of broadband applications, including multimedia services in a way to compete with wired LAN systems. Several standards such as IEEE 802.11b (providing up to $11 \mathrm{Mbps}$ ) or IEEE 802.11a (increasing speed up to $54 \mathrm{Mbps}$ ) or IEEE $802.11 \mathrm{~g}$ (increasing speed up to $108 \mathrm{Mbps}$ ) or IEEE 802.11n (increasing the speed up to $600 \mathrm{Mbps}$ ) are transforming the way we experience personal communications. There are several other standards coming up, e.g. IEEE 802.16 or IEEE 802.15G3 IEEE 802.11y that will increase capacity and range of coverage for wireless access.

\footnotetext{
${ }^{1}$ Lecturer-Omdurman Islamic University-Faculty of Eng. Sciences

${ }^{2}$ Associated Professor- University of Khartoum.
} 
IEEE 802.11a is a WLAN standard proposed by Institute of Electrical and Electronics Engineers (IEEE).Orthogonal Frequency Multiplexing (OFDM) is the chosen modulation scheme for IEEE 802.11a transmission because of its resiliency to multi path effects while achieving high data rates. The performance of OFDM systems is very sensitive to synchronization errors. A system designer must address the issues arising out of synchronization errors and devise practical methods to effectively counteract the deleterious effects within the time constraints imposed by high-speed systems like WLAN.

\section{Problem Definition}

Synchronization is a big hurdle in OFDM. The received OFDM signal is to be synchronized properly both in time and frequency. Synchronization process requires acquiring of time and frequency as well as channel estimation within a short duration of the preamble. This operation is a challenging task in the receiver.

\section{Design of the IEEE 802.11a OFDM Modem:}

The research has been started by studying the IEEE 802.11a standard and the OFDM technology. Then the research defined the issues to be study by the simulation. It has been found that the function of each block in the transmitter is completely specified in the standard but the receiver is not fully specified; only the broad functionalities and the performance requirements are specified. Also synchronization and timing is one of the big problems and challenges for receiver designer because of wireless environment and the high rate required by the standard. To define a solutions for all these problems the research reviewed a number of weight papers, researches and books which specialized in this field and then a number of roles, algorithms, methods and theorems has been used to design the receiver such as (Schmidl and Cox algorithm for packet detection, OneShot automatic gain control, Moos and Van de Beak algorithm for course frequency offset estimation Cross Correlation metric and conjugate metric for symbol timing, Van de Beek’s algorithm for fine frequency estimation, Frequency Domain Estimation (FDE) and Least Square (LS) estimation for channel estimation ). The research evaluated each algorithm by simulating a complete OFDM modem based on IEEE 802.11a standard and simulate each algorithm in the model. Mat lab has been used as a programming 
language to write the code of the simulation. Mat lab has been chosen because it is powerful and easy to use. First the mathematical model has been designed for reach part of the OFDM modem as specified in the standard document sheet and also the channel model has been designed. Most of the work was in the receiver because the objective was to solve receiving problems as mentioned before. The simulation results can be summarized the as follow:

1.packet detection:

Schimdl ad Cox proposed a simple and effective algorithm for packet detection known as Delay and Correlate. The simulation showed that the algorithm is working probably and powerful.

2. Automatic Gain Control:

The One - Shot algorithm gives good results and it is suitable with the standard.

\section{Course Frequency Offset Estimation:}

Moose proposed a maximum likelihood based estimation method for the carrier frequency offset estimation. This method calculates the correlation between the received training sequence and its repeated version in the frequency domain after the FFT operation. The main disadvantage of Moose's algorithm is that the DFT has to be calculated for both repeated symbols. Van de Beek has proposed a maximum likelihood estimation algorithm which operates on the received time domain signal. This algorithm can achieve same performance as Moose's algorithm without the DFT operation.

\section{Symbol Timing:}

The simulation showed that if the remaining frequency offset is high the conjugate metric algorithm gives better performance compared to correlation metric algorithm; on the other hand if the remaining frequency offset is small the correlation metric gives better performance.

5.Fine Frequency Offset Estimation:

The performance of frequency offset estimation algorithm has been measured in terms of the mean square error (MSE). The result indicates that the MSE is less for large value of window length (L) and periodicity (D).

6.Channel Estimation:

The results showed that the least square (LS) estimation gives better channel estimation compared to frequency domain estimation (FDE) algorithm. 
Finally, the research has been proved that the OFDM modem is very sensitive to the frequency and timing offset. The design of receiver involves critical tasks such as timing synchronization, frequency synchronization and channel estimation. Therefore, the designer of IEEE OFDM receiver should take in mined all these problems which will be a big hurdle for him.

\section{Results Discussion}

\subsection{Results of Preamble Construction}

First of all the preamble must be prepared correctly because all the receiver's tasks depends on the preamble and thus if there is any mistake in the preamble components the receiver will not detect the packet and all the modem operation will be incorrect. So in the transmitter code the preamble has been constructed and the results compared with IEEE 802.11a standard sheet. The results below are typical to the standard. Fig. (1) Show the total preamble.

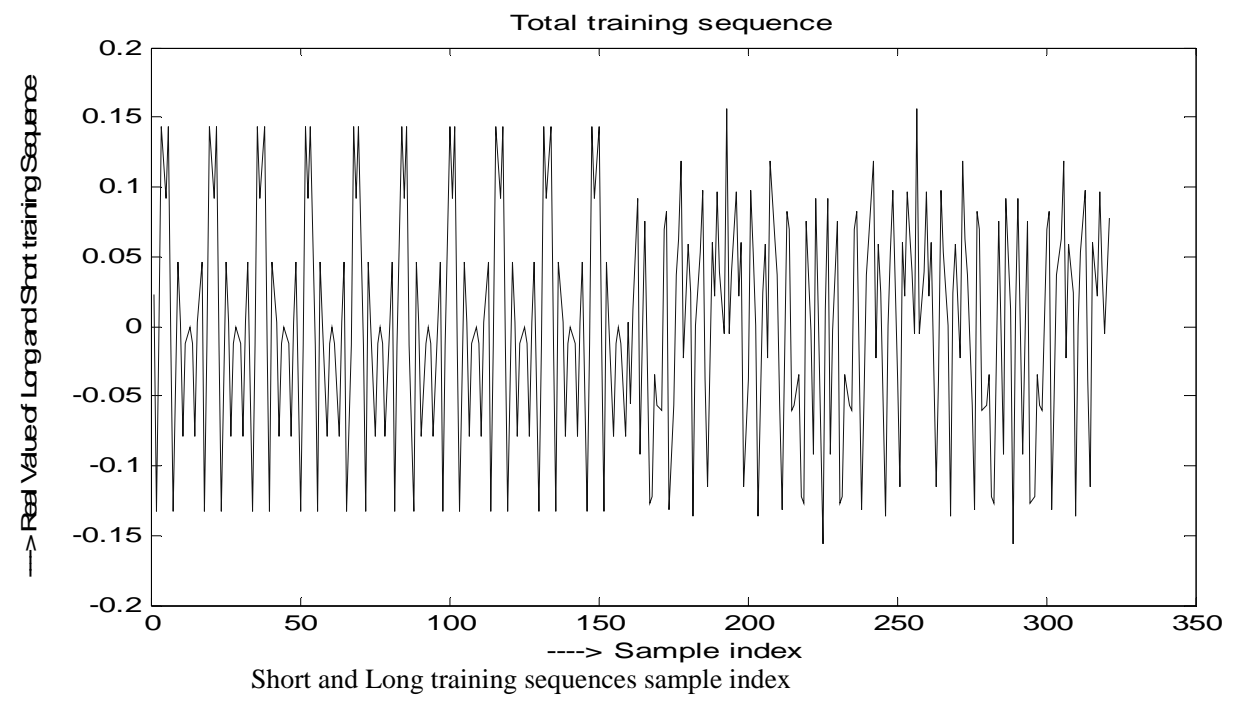

Figure (1): The preamble (Short and long training sequences) 


\subsection{Result of Packet Detection}

The arbitrary start of the packet is simulated by adding 200 zeros before start of packet. The decision metric obtained for the Delay and Correlate algorithm in Rayleigh fading channels) model having a maximum attenuation about 25db at SNR of $10 \mathrm{~dB}$ is shown in Fig (2). The actual packet starts at ' $\mathrm{n}=199$ '. The estimated timing from the metric is also 199.

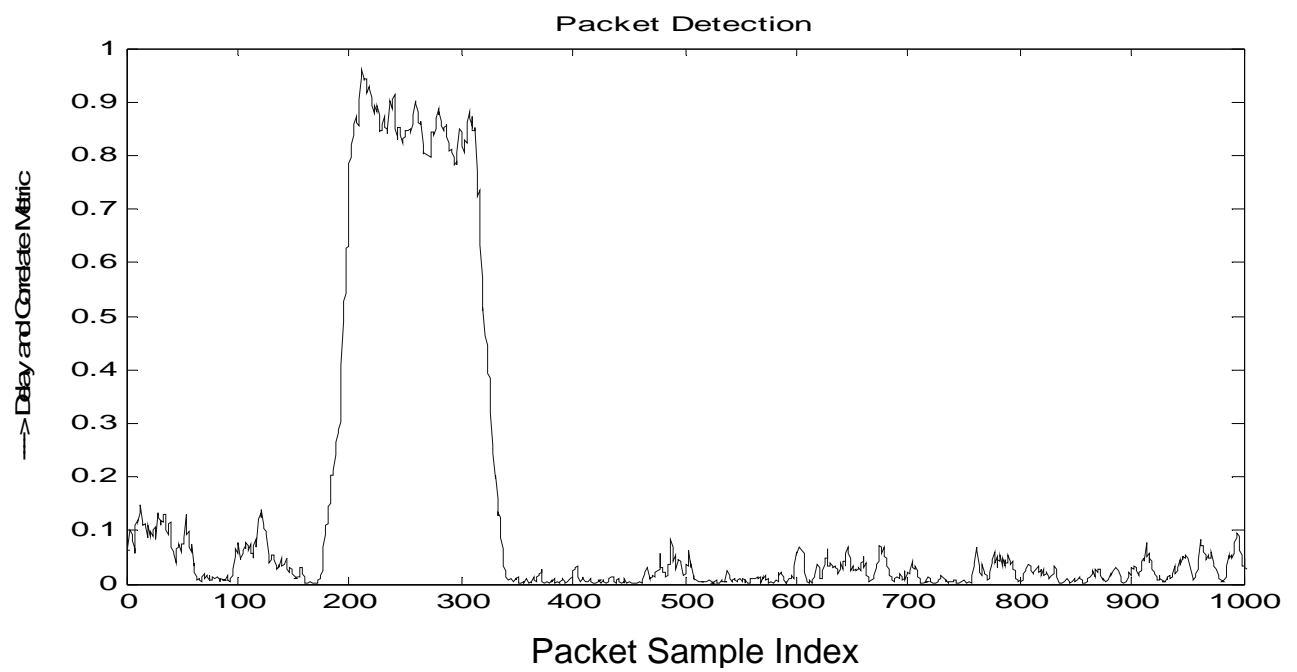

Figure (2): Packet Detection using Delay and Correlate Metric

\subsection{Results of Automatic Gain Control (AGC)}

The following figures depicts the AGC results of the received signals and the transmitted signal and we can note that the received signal effected by the channel. 


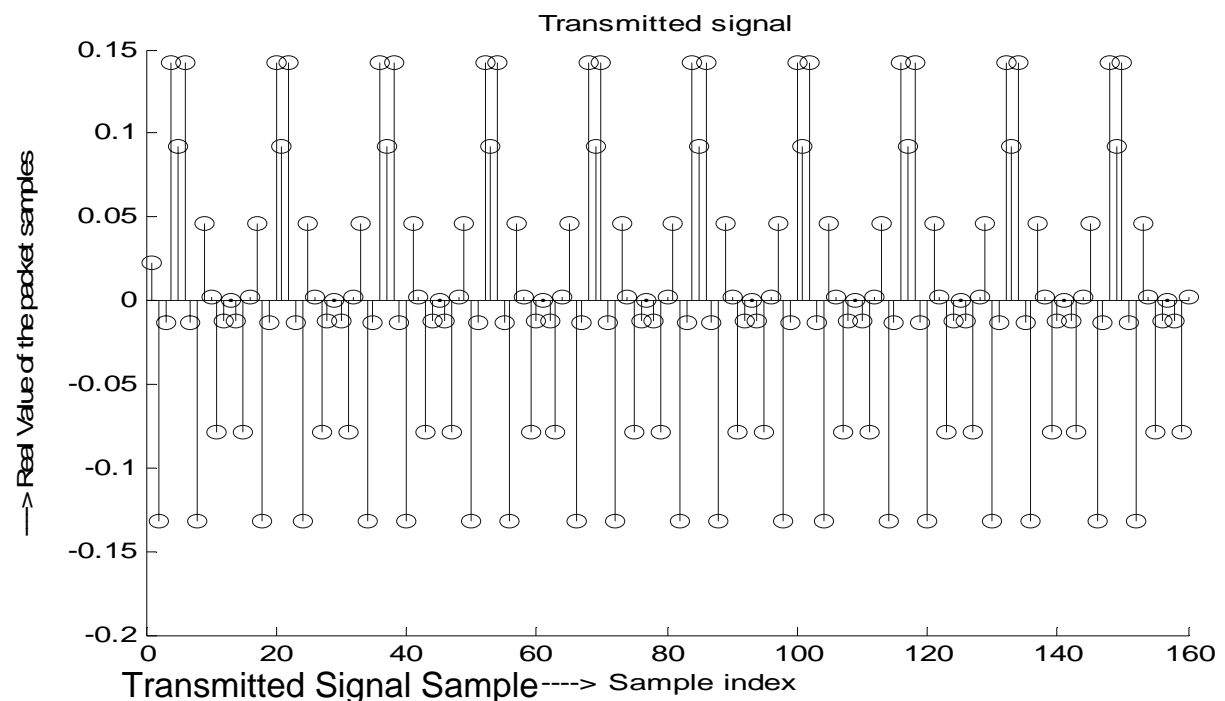

Figure (3): Transmitted signal with Automatic Gain Control applied

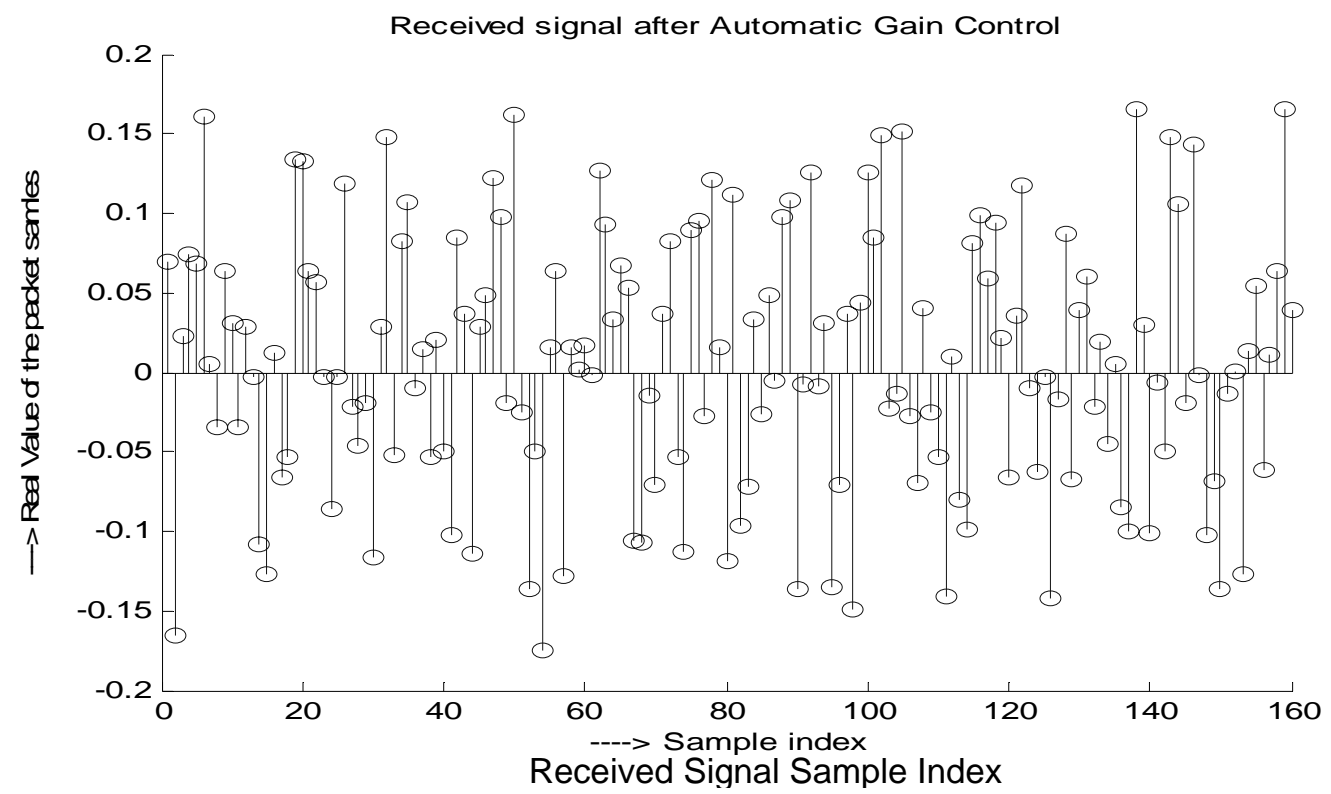

Figure (4): Received signal with Automatic Gain Control applied 


\subsection{Result of Symbol Timing}

Fig. (5) shows the Cross Correlation Function according to equation (1) in Rayleigh fading channel at SNR 10dB (assuming the remaining offset to be $100 \mathrm{KHz}$ ). The first peak obtained at 393 gives the correct symbol timing instant. Similarly the second peak corresponds to the second LTS and the smaller peak at 361 corresponds to the GI2 between the STS and the LTS. Fig. (6) shows the conjugate metric function according to equation (2) in the same channel at SNR 10dB. Four peaks are observed. Two peaks are obtained for each LTS, one at the starting point and another at the middle due to conjugate symmetry of the LTS. The first peak corresponds to the correct timing instant. In this case the correlation metric is superior to the conjugate metric.

$t_{s 1}=\arg \max _{n}\left|\sum_{k=0}^{k=L-1} r_{n+k} t_{k}^{*}\right|^{2}$

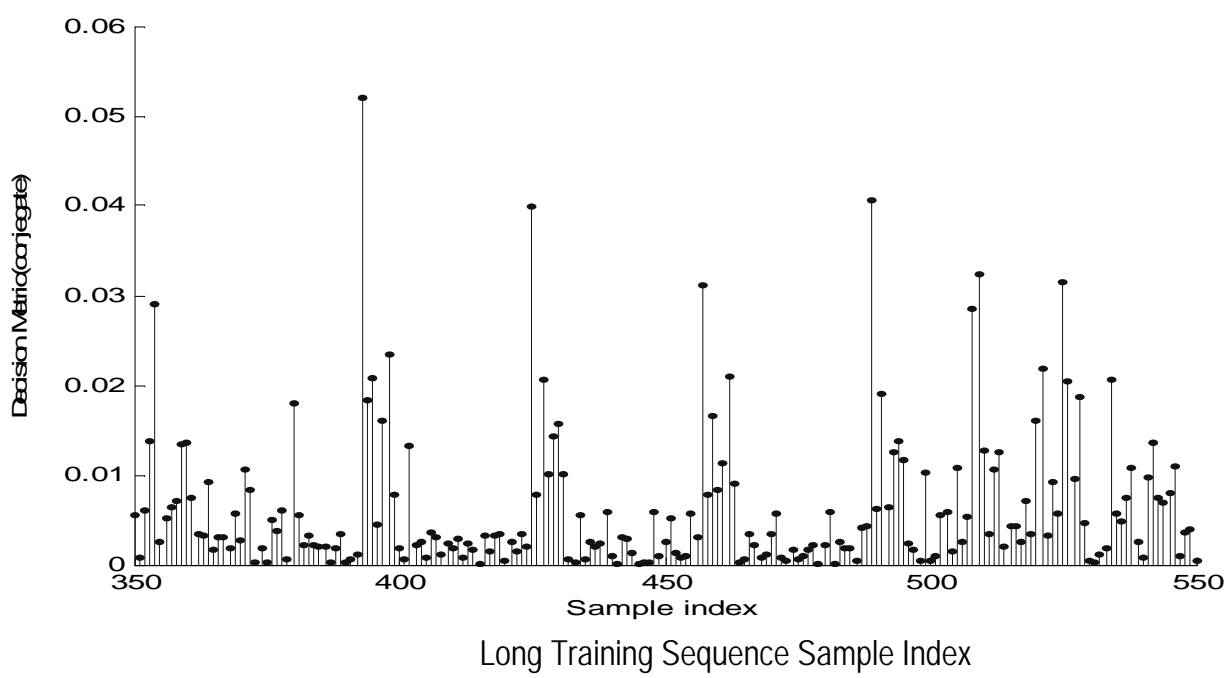

Figure (6): Long Training Sequence Detection using conjugate metric with $100 \mathrm{KHz}$ of residual frequency offset.

Fig. (7) and Fig. (8) show the results obtained assuming the remaining offset to be $200 \mathrm{KHz}$. As seen from Figs. (7) and (8), the conjugate metric is superior to the correlation metric. Hence if the remaining frequency offset is high the conjugate metric gives better performance 


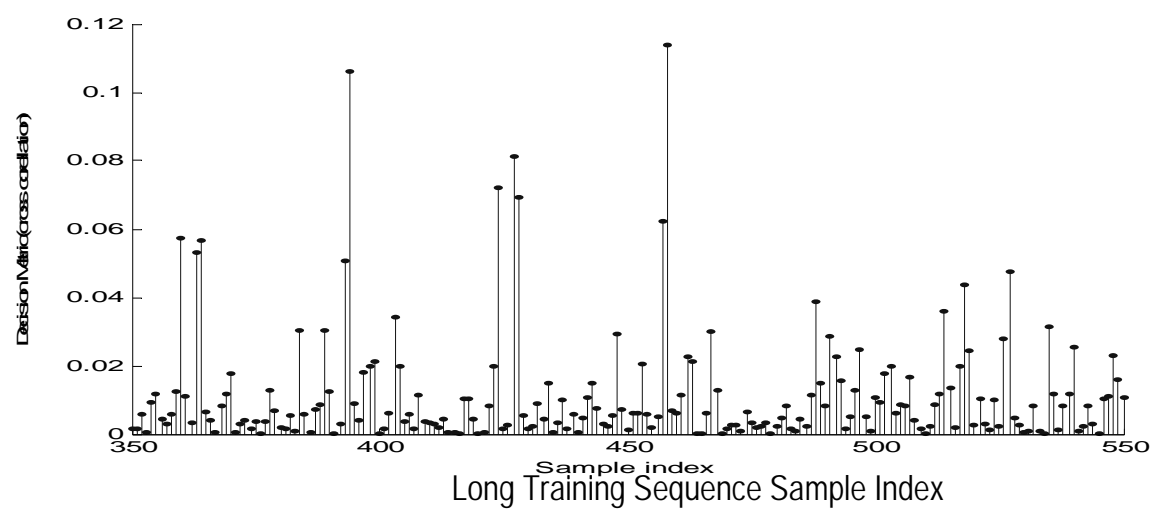

Figure (8): Long Training Sequence Detection using cross correlation

compared to correlation metric; on the other hand if the remaining frequency offset is small the correlation metric gives better performance compared to the conjugate metric.

It is to be noted that such a large residual frequency offset will not exist in actual scenario. This simulation is only to study the efficacy of the methods.

\subsection{Result of Frequency Offset Estimation:}

A frequency offset of $100 \mathrm{KHz}$ is introduced in the received signal after passing through a Rayleigh fading channel. The performance of frequency offset estimation algorithm is measured in terms of the mean square error (MSE); (where the MSE represent the deference between the actual frequency offset and the estimated frequency offset). Fig. (9) and Fig. (10) show the MSE obtained for different values of window length (L) and periodicity (D). These results indicate that the MSE is less for larger values of $L$ and $D$. The better estimate with larger window length is because of the averaging of noise over more number of samples (equation (3)) and that for larger periodicity is because of higher precision with larger D (equation (4)). But limitation on $\mathrm{D}$ comes because of reduction of the operating range for larger D. From equation (3.4), it can be seen that the maximum possible frequency error $(212 \mathrm{KHz})$ can be corrected only with values of 16 and 32 but not with values of 48 and 64 . 
Hence after the coarse frequency offset estimation (with $D=16$ or 32) and correcting the received samples with this estimated offset, fine frequency offset is performed with $\mathrm{D}=64$ using LTS. These trends clearly indicate that fine frequency offset estimation carried out with $\mathrm{D}=64$ and $\mathrm{L}=64$ will yield a much accurate frequency estimation.

$$
\begin{aligned}
& \left(f_{\in}\right)_{\text {error }}=40 \times 10^{-6} \times 5.3 \times 10^{9}=212 \mathrm{KHz} \\
& Z=e^{-j 2 \Pi f_{c} D T_{s}} \sum_{k=0}^{L-1}|x(n+k)|^{2}
\end{aligned}
$$

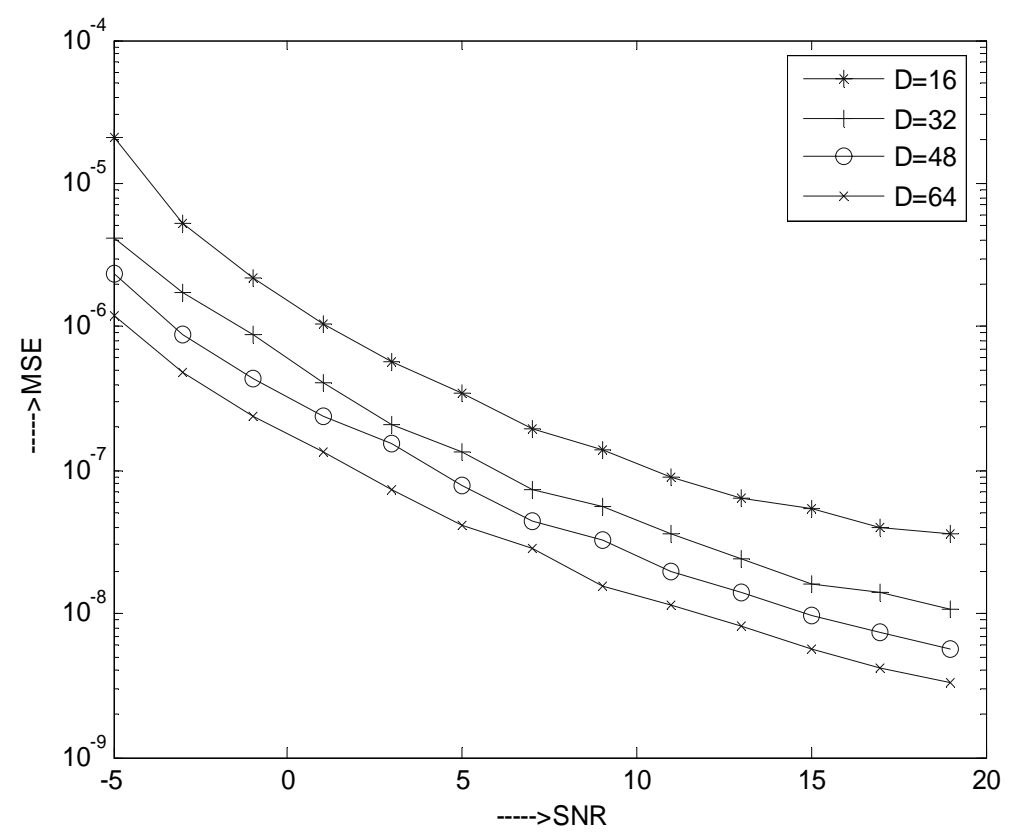

Figure (9): Mean Square Error (MSE) of Course Frequency Offset using different periodicities (D) with window length $(\mathrm{L}=$ 32). 


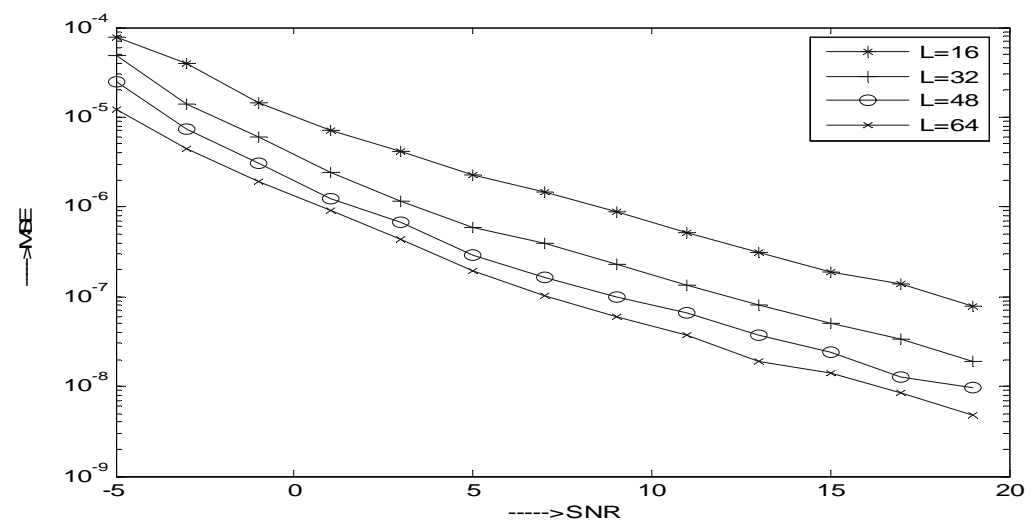

Figure (10): Mean Square Error (MSE) of Course Frequency Offset using different window lengths with periodicity $(D=16)$.

\subsection{Result of Channel Estimation:}

The performance of the Frequency Domain Channel estimation algorithm (FDC) and Least Square estimation algorithm (LS) is compared. The performance is compared in terms of normalized mean square error (NMSE) at different SNRs in an exponential decay Rayleigh channel.

$$
N M S E=\frac{\sum_{k}\left|H_{e s t}(k)-H(k)\right|^{2}}{\sum_{k}|H(k)|^{2}}
$$

Fig. (11) Shows that LS estimation gives better channel estimate compared to Frequency domain estimation algorithm. However this performance improvement is achieved at the cost of increased computational complexity. 


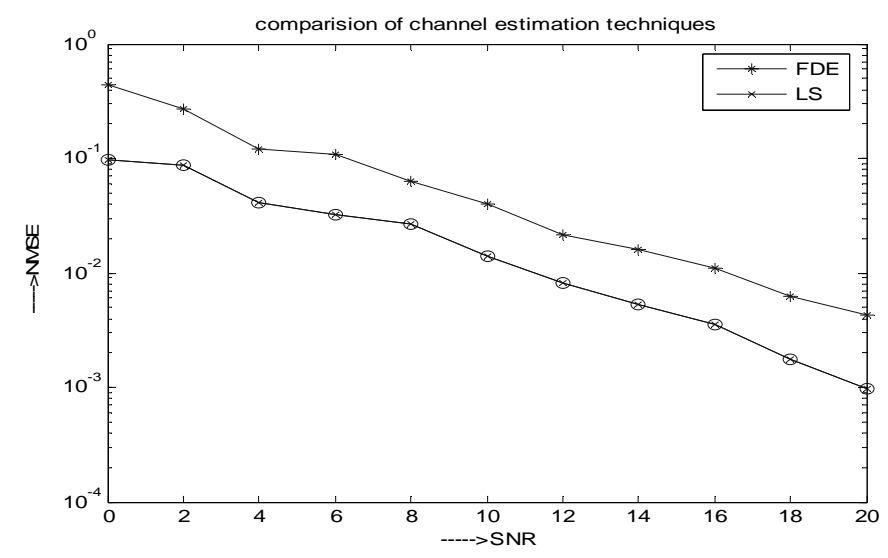

Figure (11): Comparison of channel estimation techniques (Frequency

Domain Channel (FDC) estimation and Least Square (LS) estimation for deferent Signal to Noise values (SNR).

\section{References}

[1] J.Hung Yeh et al., "WLAN Standards,” IEEE Potentials, vol. 22, Issue 4, pp. 16-22, Oct-Nov 2003.

[2] "WLAN Medium Access Control (MAC) and Physical Layer (PHY) specification,” IEEE 802.11, 1997.

[3] "WLAN Medium Access Control (MAC) and Physical Layer (PHY) specification: High - Speed Physical Layer extension in the $2.4 \mathrm{GHz}$ band," IEEE 802.11b, 1999.

[4] "WLAN Medium Access Control (MAC) and Physical Layer (PHY) specification: High - Speed Physical Layer extension in the $5 \mathrm{GHz}$ Band," IEEE 802.11a, 1999.

[5] R. Van Nee, "A new OFDM standard for high rate wireless LAN in the 5 GHz band”, Proc. of IEEE VTC99, vol.1, pp. 258-262, Sept. 1999.

[6] "WLAN Medium Access Control (MAC) and Physical Layer (PHY) specification: 
Further High Data Rate Extension in the 2.4 GHz Band,” IEEE 802.11g 2003.

[7] J. Lorincz and D. Begusic, "Physical Layer Analysis of Emerging IEEE 802.11n WLAN Standard," International Conference on Advanced Communication Technology, vol. 1, pp. 189-194, 2006

[8] J.G. Proakis and M. Salehi, Communication System Engineering, $2^{\text {nd }}$ Ed., Pearson Education Inc., India, pp. 556-560,2002.

[9] S.B. Weinster and P. M. Ebert, " Data Transmission by Frequency Division Multiplexing Using the Discrete Fourier Transform," IEEE Transaction on Communication Technology, vol. 19, No. 5, October 1971. [10] W. Henkel et. al., "The Cyclic Prefix of OFDM /DMT - An Analysis”, International Zurich Seminar on Broadband Communication, pp. 22-1-2233, 2002.

[11]J. Terry and J. Heiskala, OFDM Wireless LANs: A Theoretical and Practical Guide, Sams publishing, Indiana, 2002.

[12] N. Dinur and D. Wulich, "Peak to Average Power Ratio in High- Order OFDM,” IEEE Transaction on Communications, vol. 49, No. 6, June 2001. [13] L. Thibault and M. T. Le. "Performance Evaluation of COFDM for digital Audio Broadcasting Part I: Parametric Study," IEEE Transaction on Broadcasting, vol. 43, No. 1, pp. 64-74, March 1997.

[14] European Telecommunication standards Institute: "Digital broadcasting systems for television, sound and data services; Framing structure, channel coding and modulation for digital terrestrial television ," ETS 300744, 1996.

[15] U. Reimers, "DVB-T: the COFDM- based system for terrestrial television” Electronics and communication Engineering Journal, vol. 9, no. 1, pp. 28-32, Feb. 1997.

[16] B.R. Saltzberg, "Comparison of single-carrier and multitone digital modulation for ADSL applications” IEEE Communication Magazine, vol. 36, Issue 11, pp. 114-121, Nov. 1998.

[17] T. Zahariadis et. al., "A comparison of competing broadband in-home technology," Electronics and Communication Engineering Journal, vol. 14, Issue. 4, pp. 133-142, Aug. 2002.

[18] Timothy M. Schmidl, Donald C. Cox, "Robust frequency and timing synchronization for OFDM,” IEEE Trans. Commun., vol. 45, pp. 16131621, Dec. 1997.

[19] Byungjoon Park et. al., "A novel timing estimation method for OFDM system,” IEEE Comm. Letters, vol. 7, no. 5, pp. 239-241, May 2003. 
[20] Paul H. Moose, "A technique for orthogonal frequency division multiplexing frequency offset correction," IEEE Trans Commun., vol. 42. no. 10, pp. 2908-2914, Oct 1994.

[21] Jan-Jaap van de Beek et. al., "ML estimation of time and frequency offset in OFDM system,” IEEE Trans. Signal Processing, vol. 45, no. 7, pp. 1800-1805, July 1997.

[22] T. Pollet, M. V. Bladel, and M. Moeneclaey, "BER Sensitivity of OFDM Systems to Carrier Frequency Offset and Wiener Phase Noise," IEEE Transactions on Communications, vol. 43, Issue 2, Part 3, pp. 191193, 1995.

[23] M. Speth et. al., "Optimum Receiver Design for Wireless Broad-Band System Using OFDM - Part I,” IEEE Transaction on Communication, vol. 47, Issue 11. pp. 130-143 1999.

[24] Meng Wu, Wei-Ping Zhu, “A preamble -aided symbol and frequency synchronization scheme for OFDM system," IEEE International Symposium on Circuits and Systems, ISCAS 2005, vol. 3, pp. 2627-2630, July 1997.

[25] W.W. Kim et al. , “An AGC design of mobile cellular systems ,” IEEE Vehicular Technology Conference, vol. 3, pp. 2134-2137 Sept 2004.

[26] V. P. Gil Jimnez et. al. , "Design and Implementation of Synchronization and AGC for OFDM - based WLAN Receivers," IEEE Transaction on Consumer Electronics, vol. 50, No. 4, November 2004.

[27] G. V Rangarajan, M. R. Reghavendra and K. Giridhar, "Improved Channel Estimation for OFDM Based WLAN System,” International Conf. on Wireless Comm. Networks ICWCN - 2003, June 2003.

[28] T.S. Rappaport, Wireless Communications, Principles and Practice, $2^{\text {nd }}$ Ed. Prentice Hall Inc., India 2001.

[29] A. Pandharipande, \Principles of OFDM," IEEE Potentials, vol. 20, pp. 16-19, April

2002.

[30] www.wireless.per.nl Source: Research group of Prof. Paul Walter Baier, U. of Kaiserslautern, Germany . Accssed on 5/12/2007. [31] www.ieee.org . Accssed on 2/3/2008. 